\title{
Validation of Noninvasive Remote Dielectric Sensing System to Quantify Lung Fluid Levels
}

\author{
Teruhiko Imamura ${ }^{1, *,+} \oplus$, Wataru Gonoi ${ }^{2,+}{ }^{\oplus}$, Masakazu Hori ${ }^{1}$, Yohei Ueno ${ }^{1}$, Nikhil Narang ${ }^{3}$, Hiroshi Onoda ${ }^{1}$, \\ Shuhei Tanaka ${ }^{1}$, Makiko Nakamura ${ }^{1}$, Naoya Kataoka ${ }^{1}$, Ryuichi Ushijimaa ${ }^{1}$, Mitsuo Sobajima ${ }^{1}$, \\ Nobuyuki Fukuda ${ }^{1}$, Hiroshi Ueno ${ }^{1} \mathbb{D}$ and Koichiro Kinugawa ${ }^{1}$
}

\section{check for}

updates

Citation: Imamura, T.; Gonoi, W.;

Hori, M.; Ueno, Y.; Narang, N.;

Onoda, H.; Tanaka, S.; Nakamura, M.

Kataoka, N.; Ushijima, R.; et al.

Validation of Noninvasive Remote

Dielectric Sensing System to Quantify

Lung Fluid Levels. J. Clin. Med. 2022,

11, 164. https://doi.org/10.3390/

jcm11010164

Academic Editors: Toshiyuki Nagai

and Gaetano Ruocco

Received: 13 December 2021

Accepted: 28 December 2021

Published: 29 December 2021

Publisher's Note: MDPI stays neutral with regard to jurisdictional claims in published maps and institutional affiliations.

Copyright: (C) 2021 by the authors. Licensee MDPI, Basel, Switzerland. This article is an open access article distributed under the terms and conditions of the Creative Commons Attribution (CC BY) license (https:// creativecommons.org/licenses/by/ $4.0 /)$.
1 Second Department of Internal Medicine, University of Toyama, Toyama 9300194, Japan; masahori6059@yahoo.co.jp (M.H.); fef6ge@gmail.com (Y.U.); ohiro0203@gmail.com (H.O.); stanaka@med.u-toyama.ac.jp (S.T.); nakamuramk1979@gmail.com (M.N.); nkataoka@icloud.com (N.K.); ryuryu0702@gmail.com (R.U.); soba1126@yahoo.co.jp (M.S.); nfukuda@med.u-toyama.ac.jp (N.F.); hueno@med.u-toyama.ac.jp (H.U.); kinugawa-tky@umin.ac.jp (K.K.)

2 Department of Radiology, Graduate School of Medicine, University of Tokyo, Tokyo 1138654, Japan; gonoiw@gmail.com

3 Advocate Christ Medical Center, Oak Lawn, IL 60453, USA; nikhil.narang@gmail.com

* Correspondence: teimamu@med.u-toyama.ac.jp; Tel.: +81-76-434-2281; Fax: +81-76-434-5026

+ These authors contributed equally to this work.

\begin{abstract}
Background: The accuracy of the remote dielectric sensing (ReDS ${ }^{\mathrm{TM}}$ ) system, which is a noninvasive electromagnetic-based technology to quantify lung fluid levels, particularly among those with small body size, remains uncertain. Methods: Hospitalized patients with and without heart failure underwent assessment of lung fluid levels with ReDS and successive chest computed tomography imaging. We performed a correlation analysis of the ReDS measurement, representing lung fluid levels, and computed tomography-derived high attenuation area percentage, which also provides a spatial quantification of lung fluid level. Results: A total of 46 patients (median 76 years old, 28 men), including 28 patients with heart failure, were included. The median ReDS value was $28 \%$ (interquartile: $23 \%, 33 \%)$, and the median percentage of high attenuation area was $21.6 \%(14.4 \%$, $28.5 \%)$. ReDS values and percentage of high attenuation area were moderately correlated $(\mathrm{r}=0.65$, $p<0.001$ ), irrespective of the existence of heart failure. ReDS value independently predicted the percentage of high attenuation area seen on computed tomography $(p<0.001)$. Conclusions: The ReDS system may be a promising, noninvasive tool to quantify fluid lung levels, as validated by comparison with chest computed tomography imaging. Further studies are warranted to validate the utility and applicability of this technology to a variety of clinical scenarios.
\end{abstract}

Keywords: congestion; heart failure; hemodynamics; CT densitometry

\section{Introduction}

The significant morbidity and mortality benefit of neurohormonal antagonists in patients with chronic heart failure has been shown in large-scale randomized control trials. Comprehensive four-tier medical therapy for chronic heart failure with reduced ejection fraction including angiotensin-neprilysin inhibitors, beta-blockers, mineralocorticoid receptor antagonists, and sodium-glucose cotransporter 2 inhibitors can offer dramatic additional clinical risk reduction compared to angiotensin converting enzyme inhibitors and betablockers alone [1]. Promising data have also recently emerged demonstrating the benefit of both angiotensin-neprilysin inhibitors and sodium-glucose cotransporter 2 inhibitors in patients with heart failure and preserved ejection fraction [2,3].

Nevertheless, the management of residual pulmonary congestion is an important treatment goal given its impact on patient quality of life [4]. However, given the lack of a gold-standard to accurately assess lung fluid levels, precise management of pulmonary 
congestion remains a clinical challenge. Chest computed tomography $(\mathrm{CT})$ can quantify the presence of pleural and interstitial lung fluid, but is cumbersome for monitoring, poses a risk of radiation, and comprises a highly complex methodology.

Recently, a remote dielectric sensing system (ReDS ${ }^{\mathrm{TM}}$, Sensible Medical Innovations Ltd., Netanya, Israel), a noninvasive electromagnetic-based technology to quantify lung fluid volumes, was introduced for clinical use [5]. A preliminary analysis observed a significant correlation between ReDS values and lung fluid amount measured by highresolution CT [6]. Additional contemporary analyses have also shown the benefit of ReDS measurements in the guidance of heart failure management [7,8]. However, these studies were conducted in Western countries and did not include subjects with smaller body sizes. In this study, we investigated the correlation between ReDS values and lung fluid levels, measured by chest CT imaging to validate ReDS quantification of pulmonary congestion in a broad range of body sizes.

\section{Methods}

\subsection{Participant Selection}

In this prospective study, hospitalized patients with and without heart failure received ReDS measurements following clinical stabilization. Chest CT imaging was performed on the same day following the ReDS measurements. Patients who were unable to have ReDS measurements taken, including those with intrathoracic devices such as permanent pacemakers, or pulmonary lesions including lung malignancies and pneumonia, were excluded. Informed consent was obtained from all participants beforehand. The institutional ethical review board approved the study protocol (MTK2020007).

\subsection{ReDS System}

The ReDS system estimates lung fluid levels and degree of pulmonary congestion under natural breathing conditions [5]. ReDS employs low-power electromagnetic signals emitted between two sensors embedded on a wearable device (Figure 1). The analyzed signals reflect the dielectric properties of the lung portion between the two sensors. The dielectric coefficients of a material are represented by a frequency-dependent number describing its interaction with electromagnetic energy, including the degrees of reflection, absorption, and transmission of the energy. Given that water has a high dielectric coefficient and air has a low one, the dielectric coefficient of tissue is determined predominantly by its fluid content. The normal range for the ReDS value, as proposed by the product's manufacturer, is between $20 \%$ and $35 \%$.

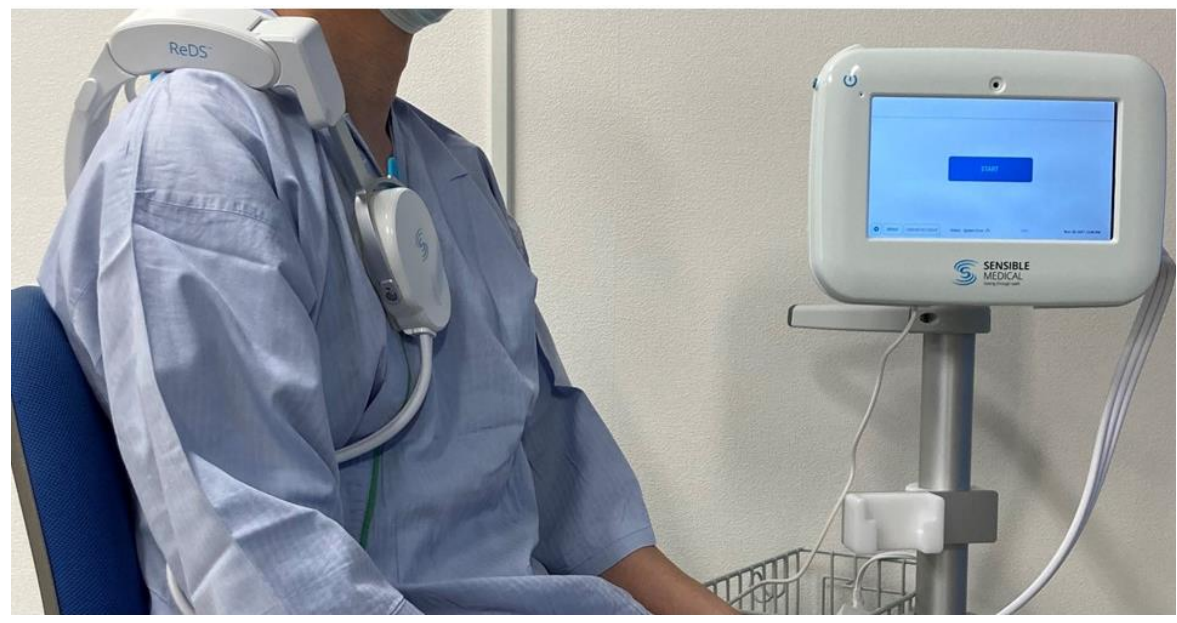

Figure 1. A ReDS system consisting of a monitor and a sensor unit. 


\subsection{CT Image Acquisition}

All patients underwent $\mathrm{CT}$ scan examinations in the supine position at full inspiration without intravenous contrast. CT images were acquired using a dual-source CT unit (SOMATOM Force scanner, Siemens Healthineers, Erlangen, Germany). The scanning parameters were as follows: tube voltage, $120 \mathrm{kVp}$; tube current, controlled by automated tube current modulation; matrix size, $512 \times 512$; pixel spacing, $0.625 \mathrm{~mm} \times 0.625 \mathrm{~mm}$. CT images of 5-mm slice thickness were reconstructed using lung reconstruction kernels.

\section{Automated Volume Analysis of the CT Images}

A commercially available CT imaging analysis workstation, the Synapse VINCENT Ver 6.4.0003 (Fujifilm Medical Systems, Tokyo, Japan), was used for volumetric analysis of hyperattenuated lungs $[9,10]$.

First, an expert diagnostic radiologist who was blinded to the clinical data including ReDS values evaluated the imaging to rule out the presence of lung lesions other than pulmonary congestion and mild emphysema, along with those with pneumonias and neoplasms, who were excluded.

Second, using a lung analysis tool for automatic whole lung extraction from the chest, CT images were taken excluding the thoracic wall, mediastinum, large vessels, pleural effusion, and airways (from the trachea to tertiary bronchi) (Figure 2A-C).

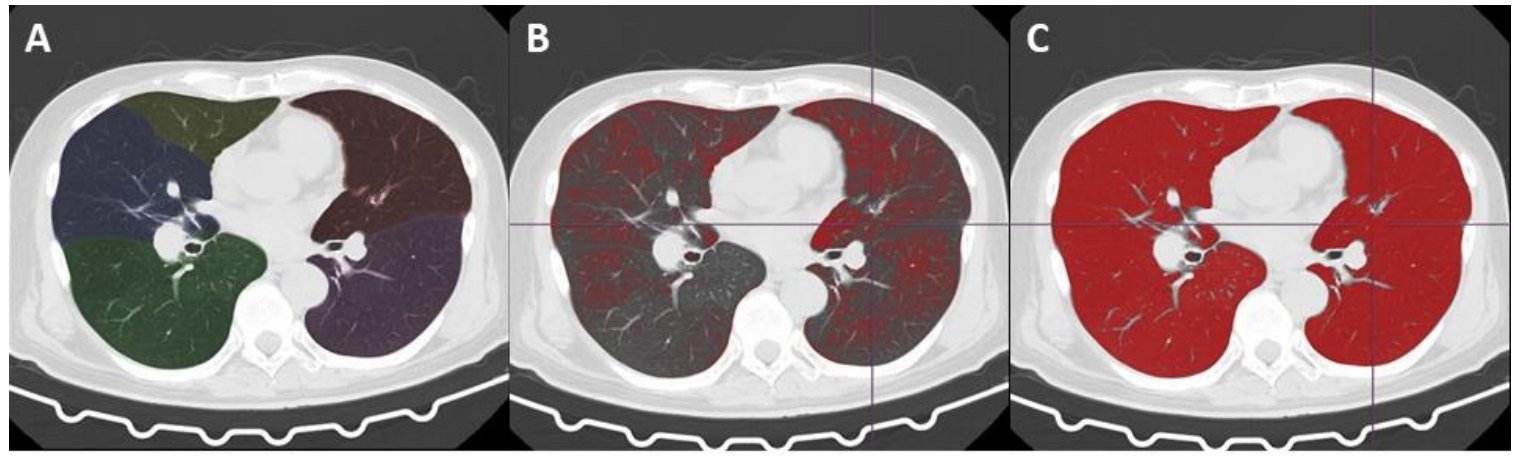

Figure 2. Automated volume analysis of the CT images. Chest computed tomography (CT) image of a 73-year-old man with suspected pulmonary hypertension. Automatically extracted and segmented lungs and lobes are color-coded (A). Red-painted areas indicate lung parenchyma with and under $-950 \mathrm{HU}$ (B) and $-750 \mathrm{HU}$ (C), which amounted to $21.7 \%$ and $91.6 \%$ of the whole lung (whole volume, $6456.6 \mathrm{~mL}$; average density, $-885.1 \mathrm{HU})$, respectively. Therefore, this patient's \%high attenuation area (from $-749 \mathrm{HU}$ to $0 \mathrm{HU}$ ) was calculated as $8.4 \%$ of the whole lung.

Third, a threshold-based volumetric CT analysis was performed to estimate the volume of hyperattenuated lung, which indicates pulmonary congestion, and its ratio to whole lung volume.

Although there is no established CT value threshold for normally attenuated lung and lungs with pulmonary congestion, based on several past threshold-based analyses for various lung diseases, we set the lower and upper limit CT value for a normal lung at $-950 \mathrm{HU}$ and different $\mathrm{CT}$ values to within the range from -800 to -500 , respectively [11-15].

A past study reported that early pulmonary congestion and edema due to left-sided heart disease caused a rise in CT values, prominently in the range between -750 and $-650 \mathrm{HU}$, suggesting that $-750 \mathrm{HU}$ is a reasonable candidate threshold for distinguishing normal lung and pulmonary congestion [16].

Finally, we defined the threshold for the present study as follows: the whole lung, from $-1000 \mathrm{HU}$ to $0 \mathrm{HU}$; low attenuation area (emphysematous lung), $-1000 \mathrm{HU}$ to $950 \mathrm{HU}$; normal attenuation area (normal lung), from $-949 \mathrm{HU}$ to $-750 \mathrm{HU}$; high attenuation area (edematous lung), from $-749 \mathrm{HU}$ to $0 \mathrm{HU}$. 
In the process of lung extraction and threshold-based analysis, the volumes and mean CT values were available for each lung portion. The radiologist checked all the processed images and measured values, and confirmed that the analyses had been performed correctly.

\subsection{Statistical Methods}

Continuous variables are presented as medians and interquartiles. Categorical variables are presented as numbers and percentages. The correlation between ReDS values and \%high attenuation area (volumetric ratio of high attenuation area to the whole lung) was assessed using Pearson's correlation. Linear regression analyses were conducted to investigate the impact of baseline characteristics, including ReDS values, on the \%high attenuation area. All analyses were performed in SPSS Statistics 23.0 software (IBM Corp, Armonk, NY, USA), and two-tailed $p$ values less than 0.05 were assumed significant.

\section{Results}

\subsection{Baseline Characteristics}

A total of 46 hospitalized patients were included (Table 1). The median age was 76 (73, $84)$ years old, and 28 (61\%) subjects were men. The median body mass index was 21.6 (19.7, 26.0). The distribution of ReDS values and \%high attenuation area are shown in Figure 3A,B. The median ReDS value was $28 \%$ (23\%, 33\%), and the median \%high attenuation area was $21.6 \%(14.4 \%, 28.5 \%)$.

A.

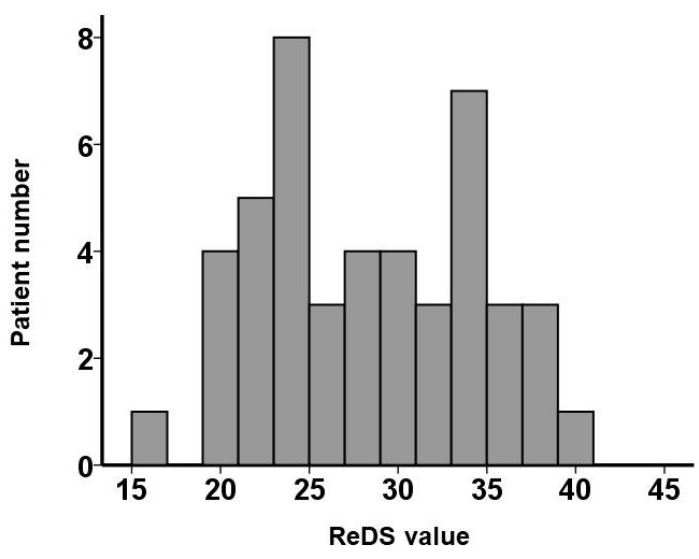

B.

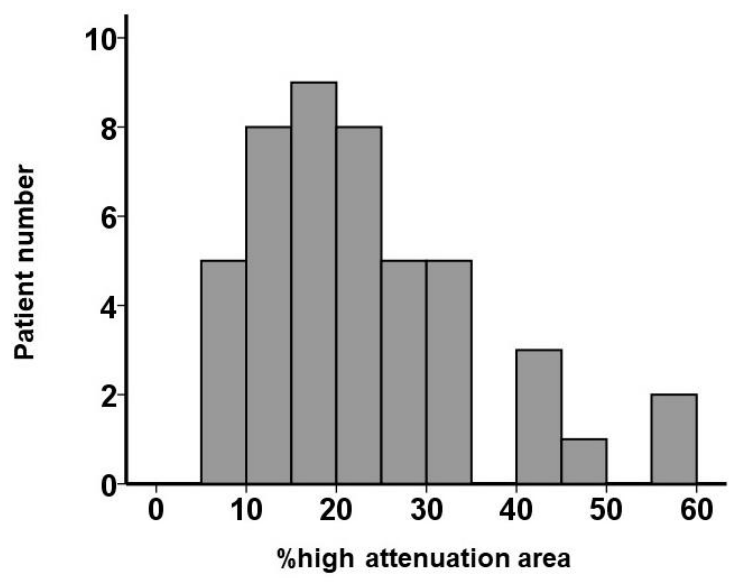

Figure 3. Distribution of ReDS value (A) and \%high attenuation area (B). 
Table 1. Baseline characteristics.

\begin{tabular}{|c|c|c|c|c|}
\hline & Total $(\mathrm{N}=46)$ & $\begin{array}{c}\text { Heart } \\
\text { Failure }(N=28)\end{array}$ & $\begin{array}{l}\text { Nonheart Failure } \\
\quad(\mathbf{N}=18)\end{array}$ & $p$ Value \\
\hline \multicolumn{5}{|l|}{ Demographics } \\
\hline Age, years & $76(73,84)$ & $75(72,81)$ & $79(74,86)$ & 0.86 \\
\hline Men & $28(61 \%)$ & $16(57 \%)$ & $12(67 \%)$ & 0.37 \\
\hline Body height, $\mathrm{cm}$ & $159(150,167)$ & $161(151,168)$ & $157(149,166)$ & 0.45 \\
\hline Body mass index, $\mathrm{kg} / \mathrm{m}^{2}$ & $21.6(19.7,26.0)$ & $20.8(19.4,23.9)$ & $23.8(21.5,26.7)$ & 0.087 \\
\hline \multicolumn{5}{|l|}{ Comorbidity } \\
\hline Hypertension & $33(72 \%)$ & $23(82 \%)$ & $10(56 \%)$ & 0.054 \\
\hline Dyslipidemia & $26(57 \%)$ & $17(61 \%)$ & $9(50 \%)$ & 0.34 \\
\hline Diabetes mellitus & $19(41 \%)$ & $12(43 \%)$ & $7(39 \%)$ & 0.52 \\
\hline Chronic kidney disease & $24(52 \%)$ & $14(50 \%)$ & $10(56 \%)$ & 0.47 \\
\hline History of stroke & $8(17 \%)$ & $5(18 \%)$ & $3(17 \%)$ & 0.92 \\
\hline Coronary heart disease & $7(15 \%)$ & $2(7 \%)$ & $5(28 \%)$ & 0.057 \\
\hline Atrial fibrillation & $9(20 \%)$ & $7(25 \%)$ & $2(11 \%)$ & 0.25 \\
\hline \multicolumn{5}{|l|}{ Echocardiography } \\
\hline Left atrial diameter, $\mathrm{mm}$ & $41(36,46)$ & $40(33,46)$ & $43(41,46)$ & 0.29 \\
\hline $\begin{array}{l}\text { Left ventricular end-diastolic diameter, } \\
\mathrm{mm}\end{array}$ & $48(45,55)$ & $51(46,56)$ & $46(43,49)$ & $0.010 *$ \\
\hline Left ventricular ejection fraction, $\%$ & $55(47,67)$ & $51(38,59)$ & $67(58,75)$ & $<0.001 *$ \\
\hline Mild or greater aortic regurgitation & $14(30 \%)$ & $11(39 \%)$ & $3(17 \%)$ & 0.095 \\
\hline Mild or greater mitral regurgitation & $23(50 \%)$ & $17(61 \%)$ & $6(33 \%)$ & 0.065 \\
\hline Mild or greater tricuspid regurgitation & $20(43 \%)$ & $13(46 \%)$ & $7(39 \%)$ & 0.42 \\
\hline \multicolumn{5}{|l|}{ Laboratory data } \\
\hline Hemoglobin, $\mathrm{g} / \mathrm{dL}$ & $11.9(10.3,13.4)$ & $11.9(10.8,13.1)$ & $11.9(10.0,14.4)$ & 0.41 \\
\hline Serum albumin, $\mathrm{g} / \mathrm{dL}$ & $3.6(3.1,3.9)$ & $3.6(3.3,4.0)$ & $3.6(2.8,3.9)$ & 0.63 \\
\hline Serum sodium, mEq/L & $139(137,142)$ & $140(138,142)$ & $139(137,141)$ & 0.42 \\
\hline $\mathrm{eGFR}, \mathrm{mL} / \mathrm{min} / 1.73 \mathrm{~m}^{2}$ & $47.1(31.1,63.2)$ & $47.5(29.3,63.2)$ & $45.1(31.1,63.0)$ & 0.61 \\
\hline Plasma B-type natriuretic peptide, pg/mL & $207(53,501)$ & $398(179,834)$ & $42(16,152)$ & $<0.001 *$ \\
\hline \multicolumn{5}{|l|}{ Medication } \\
\hline Beta-blocker & $19(41 \%)$ & $14(50 \%)$ & $5(28 \%)$ & 0.12 \\
\hline Renin-angiotensin system inhibitor & $24(52 \%)$ & $18(64 \%)$ & $6(33 \%)$ & 0.040 * \\
\hline Mineralocorticoid receptor antagonist & $10(22 \%)$ & $6(21 \%)$ & $4(22 \%)$ & 0.61 \\
\hline Loop diuretics & $14(32 \%)$ & $8(29 \%)$ & $6(33 \%)$ & 0.49 \\
\hline ReDS value, \% & $28(23,33)$ & $28(23,34)$ & $25(24,31)$ & 0.21 \\
\hline$\%$ high attenuation area, $\%$ & $21.6(14.4,28.5)$ & $22.6(17.5,30.0)$ & $17.5(12.1,28.1)$ & 0.10 \\
\hline
\end{tabular}

eGFR, estimated glomerular filtration ratio; ReDS, remote dielectric sensing. ${ }^{*} p<0.05$. Continuous variables are presented as median and interquartile and compared between the two groups using Mann-Whitney $U$ test. Categorical variables are presented as numbers and percentages and compared between the two groups using Fischer's exact test.

Within the overall cohort, 28 patients had heart failure. Other reasons for admission were ischemic heart disease, pulmonary hypertension, and acute kidney injury. Heart failure patients had a worse ventricular function and higher plasma B-type natriuretic peptide (Table 1). The ReDS value and \%high attenuation area tended to be higher in the heart failure group (Table 1).

\subsection{ReDS Value and \%High Attenuation Area}

ReDS values moderately correlated with \%high attenuation area in the overall cohort $(\mathrm{r}=0.65, p<0.001$; Figure 4A), heart failure cohort $(\mathrm{r}=0.66, p<0.001$; Figure 4B), and nonheart failure cohort $(\mathrm{r}=0.53, p=0.024$; Figure $4 \mathrm{C})$. A moderate correlation was observed among those with body height $\leq 155 \mathrm{~cm}(\mathrm{r}=0.76, p=0.001, \mathrm{~N}=16$; Figure $4 \mathrm{D})$ and those with body height $>155 \mathrm{~cm}(\mathrm{r}=0.60, p<0.001 ; \mathrm{N}=31$; Figure 4E). A representative CT image with elevated ReDS value and \%high attenuation area ( $34 \%$ and $56.9 \%$, respectively) is displayed in Figure 5. Yellow arrows indicate bilateral pulmonary congestion. 
The ReDS value was an independent predictor of \%high attenuation area after adjusting for two potential confounders (body mass index and plasmas B-type natriuretic peptide) $(p<0.001$; Table 2).

Table 2. Association between \%high attenuation area and clinical variables including ReDS value.

\begin{tabular}{|c|c|c|c|c|c|}
\hline & \multicolumn{2}{|c|}{ Univariate Analysis } & \multicolumn{3}{|c|}{ Multivariate Analysis } \\
\hline & Beta Value & $p$ Value & Beta Value & $p$ Value & VIF \\
\hline Age, years & 0.168 & 0.24 & & & \\
\hline Body mass index, $\mathrm{kg} / \mathrm{m}^{2}$ & -0.557 & 0.23 & -0.461 & 0.19 & 1.050 \\
\hline Left ventricular ejection fraction, $\%$ & -0.127 & 0.22 & & & \\
\hline Mild or greater mitral regurgitation & 4.191 & 0.25 & & & \\
\hline Serum albumin, $\mathrm{g} / \mathrm{dL}$ & -3.855 & 0.15 & & & \\
\hline $\mathrm{eGFR}, \mathrm{mL} / \mathrm{min} / 1.73 \mathrm{~m}^{2}$ & -0.055 & 0.48 & & & \\
\hline $\begin{array}{l}\text { Plasma B-type natriuretic peptide, } \\
\mathrm{pg} / \mathrm{mL}\end{array}$ & 0.007 & $0.007^{*}$ & 0.004 & 0.12 & 1.147 \\
\hline ReDS value, $\%$ & 1.301 & $<0.001 *$ & 1.196 & $<0.001 *$ & 1.098 \\
\hline
\end{tabular}

eGFR, estimated glomerular filtration ratio; ReDS, remote dielectric sensing; VIF, variance inflation factor. * $p<0.05$ by linear regression analysis. Variables that are considered clinically potential confounders were included in the multivariate analysis after excluding their multicollinearity with $\mathrm{VIF}<5.0$.
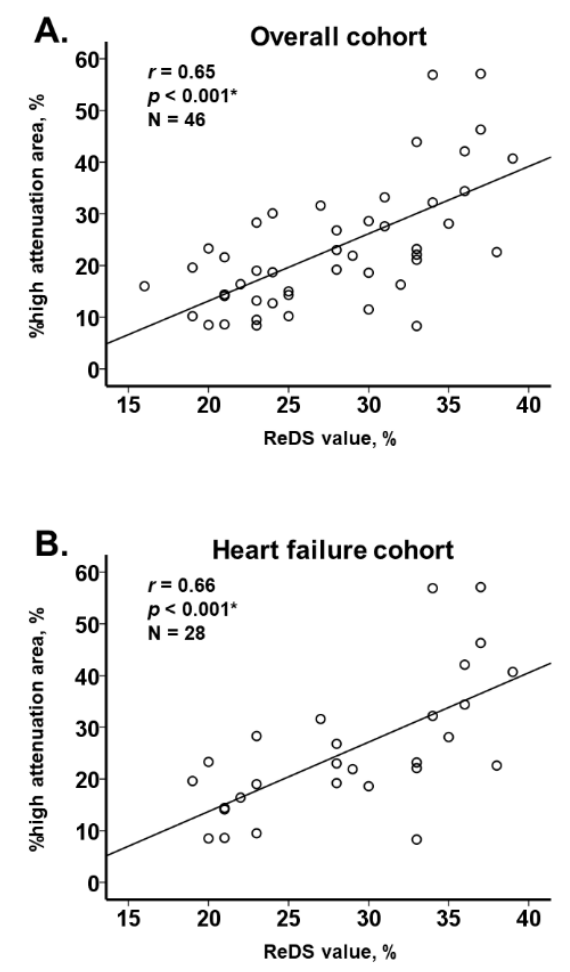

Figure 4. Cont. 

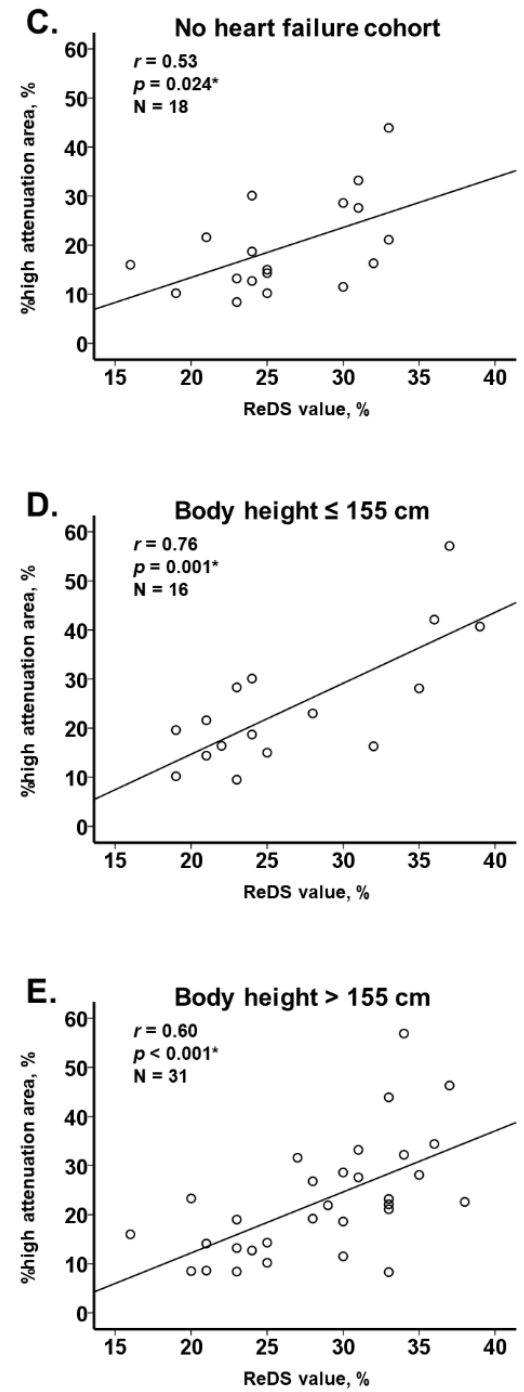

Figure 4. Correlation between ReDS value and \%high attenuation area among all cohort (A), those with and without heart failure $(\mathbf{B}, \mathbf{C})$, and those with and without body weight $\leq 155 \mathrm{~cm}(\mathbf{D}, \mathbf{E})$. * $p<0.05$ by Pearson's correlation coefficient.

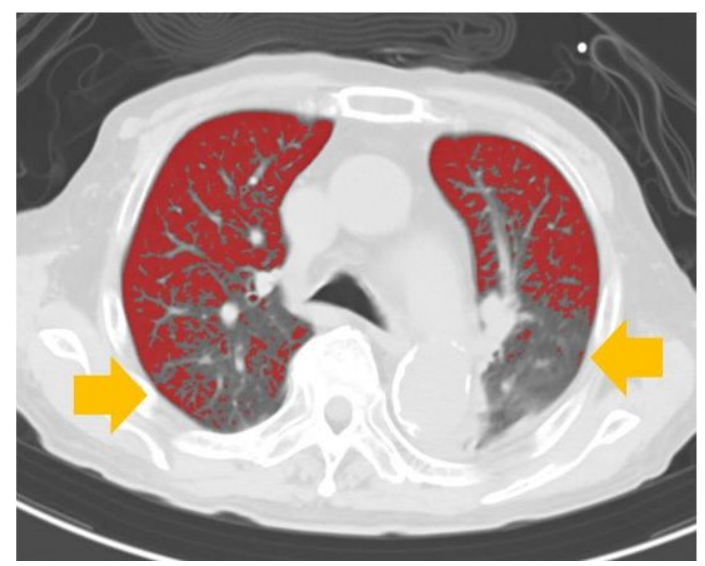

Figure 5. A representative case of pulmonary congestion. Chest CT image of a 75-year-old man with aortic stenosis and heart failure showing 34\% ReDS and 56.9\% high attenuation area. Yellow arrows in the bilateral lungs indicate hyperattenuated areas due to pulmonary congestion. 


\section{Discussion}

In this study, we observed a moderate positive correlation between the ReDS value and \%high attenuation area, both of which estimate lung fluid amount, irrespective of the presence of heart failure and body size.

\subsection{Conventional Methodologies to Assess Pulmonary Congestion}

Early and accurate methodologies to assess pulmonary congestion for patients prior to developing dyspnea are needed to curb the potential for rehospitalization due to heart failure, which is independently associated with a worse clinical prognosis.

Physical examination and chest $X$-ray are conventional and practical tools to assess for pulmonary congestion. However, they require expert technique and are often inaccurate, with intracardiac pressures often being underestimated in patients with chronic heart failure [17]. Plasma B-type natriuretic peptide is used as a surrogate of clinical risk in patients with chronic heart failure. The value reflects ventricular wall stress and distension, and does not always accompany pulmonary congestion. Various modifiers, including the presence of obesity, baseline renal function, age, and sex, may affect the interpretation of the absolute value [18]. Echocardiography may offer indirect surrogates to estimate filling pressures but does not universally quantify the degree of pulmonary congestion.

\subsection{ReDS versus Invasive Hemodynamic Measurement}

Right heart catheterization is the most accurate instantaneous method to quantify the intracardiac hemodynamics of heart failure [19]. However, patients with low cardiac output may, at times, have no pulmonary congestion despite incremental intracardiac pressures. Furthermore, given its invasiveness, right heart catheterization may not be feasible under certain circumstances. An intermediary is needed, and given its high sensitivity, the ReDS system may be a useful screening tool in patients with suspected pulmonary congestion who would require further intensive assessment including right heart catheterization [20].

\subsection{ReDS System and Chest CT}

We demonstrated a moderate correlation between the ReDS value and \%high attenuation area calculated by chest CT, irrespective of body size. Patients with smaller body sizes, particularly those with a height $\leq 155 \mathrm{~cm}$, were not included in previous studies [6-8].

$\mathrm{CT}$ s require radiation exposure and expert interpretation. The ReDS system has the advantages of being noninvasive, simple to use and providing data which is easy to interpret, all of which allow repeated assessments of pulmonary congestion on follow-up, screening, and discharge.

In contrast, chest CT can visually assess intrathoracic abnormalities in detail, including pneumonic processes and pleural effusions. Chest CT can be applied irrespective of thoracic anatomical abnormalities. The ReDS system is based on the hypothesis that pulmonary congestion is uniform among the whole lung, and focuses specifically on the right lung field [5]. The ReDS system might be inappropriate to assess heterogeneously distributed pulmonary congestion.

As a whole, \%high attenuation areas were relatively lower than ReDS value. We might have to consider the difference of breathing situation between the two modalities. Patients held their breath at full inspiration during the CT imaging scan. In contrast, ReDS values are measured during natural breathing. The lung is filled with air at full inspiration, and the \%high attenuation area might be relatively underestimated compared to that obtained during natural breathing.

\subsection{Study Limitations}

The present study was a proof-of-concept, using the ReDS system for the first time before commercial marketing in Japan. We enrolled only a small cohort. Patients received ReDS measurements after hemodynamic stabilization. The applicability of our findings to those with unstable hemodynamics with significant pulmonary congestion remains 
uncertain. Further studies are warranted to validate the applicability of this technology to various other clinical situations. At present, we recommend the use of ReDS during screening and follow-up. In critical situations, we recommend multimodalities instead of ReDS alone. We performed linear regression analyses considering that certain clinical conditions may have affected the ReDS values. However, there may have been other, uninvestigated confounders.

\section{Conclusions}

The ReDS system is a promising, noninvasive and easy-to-measure tool to quantify fluid lung fluid levels.

Author Contributions: Conceptualization, T.I.; Data curation, M.H., Y.U., H.O., S.T., M.N., N.K., R.U., M.S., N.F. and H.U.; Formal analysis, T.I. and W.G.; Investigation, T.I.; Project administration, T.I.; Software, T.I.; Supervision, K.K.; Visualization, T.I. and W.G.; Writing—original draft, T.I.; Writingreview \& editing, W.G. and N.N. All authors have read and agreed to the published version of the manuscript.

Funding: This research received no external funding.

Institutional Review Board Statement: The study was conducted in accordance with the Declaration of Helsinki, and approved by the Ethics Committee of University of Toyama (MTK2020007, 3 March 2021).

Informed Consent Statement: Informed consent was obtained from all subjects involved in the study.

Data Availability Statement: Data are available upon appropriate request.

Conflicts of Interest: All authors have no conflict of interest.

\section{References}

1. Vaduganathan, M.; Claggett, B.L.; Jhund, P.S.; Cunningham, J.W.; Pedro Ferreira, J.; Zannad, F.; Packer, M.; Fonarow, G.C.; McMurray, J.J.V.; Solomon, S.D. Estimating lifetime benefits of comprehensive disease-modifying pharmacological therapies in patients with heart failure with reduced ejection fraction: A comparative analysis of three randomised controlled trials. Lancet 2020, 396, 121-128. [CrossRef]

2. Pfeffer, M.A.; Shah, A.M.; Borlaug, B.A. Heart Failure With Preserved Ejection Fraction In Perspective. Cir. Res. 2019, 124, 1598-1617. [CrossRef] [PubMed]

3. Anker, S.D.; Butler, J.; Filippatos, G.; Ferreira, J.P.; Bocchi, E.; Bohm, M.; Brunner-La Rocca, H.P.; Choi, D.J.; Chopra, V.; ChuquiureValenzuela, E.; et al. Empagliflozin in Heart Failure with a Preserved Ejection Fraction. N. Engl. J. Med. 2021, 385, $1451-1461$. [CrossRef] [PubMed]

4. $\quad$ Girerd, N.; Seronde, M.F.; Coiro, S.; Chouihed, T.; Bilbault, P.; Braun, F.; Kenizou, D.; Maillier, B.; Nazeyrollas, P.; Roul, G.; et al. Integrative Assessment of Congestion in Heart Failure Throughout the Patient Journey. JACC Heart Fail. 2018, 6, $273-285$. [CrossRef] [PubMed]

5. Amir, O.; Rappaport, D.; Zafrir, B.; Abraham, W.T. A novel approach to monitoring pulmonary congestion in heart failure: Initial animal and clinical experiences using remote dielectric sensing technology. Congest. Heart Fail. 2013, 19, 149-155. [CrossRef] [PubMed]

6. Amir, O.; Azzam, Z.S.; Gaspar, T.; Faranesh-Abboud, S.; Andria, N.; Burkhoff, D.; Abbo, A.; Abraham, W.T. Validation of remote dielectric sensing (ReDS) technology for quantification of lung fluid status: Comparison to high resolution chest computed tomography in patients with and without acute heart failure. Int. J. Cardiol. 2016, 221, 841-846. [CrossRef] [PubMed]

7. Amir, O.; Ben-Gal, T.; Weinstein, J.M.; Schliamser, J.; Burkhoff, D.; Abbo, A.; Abraham, W.T. Evaluation of remote dielectric sensing (ReDS) technology-guided therapy for decreasing heart failure re-hospitalizations. Int. J. Cardiol. 2017, 240, $279-284$. [CrossRef] [PubMed]

8. Lala, A.; Barghash, M.H.; Giustino, G.; Alvarez-Garcia, J.; Konje, S.; Parikh, A.; Ullman, J.; Keith, B.; Donehey, J.; Mitter, S.S.; et al. Early use of remote dielectric sensing after hospitalization to reduce heart failure readmissions. ESC Heart Fail. 2021, 8, 1047-1054. [CrossRef] [PubMed]

9. Kitano, M.; Iwano, S.; Hashimoto, N.; Matsuo, K.; Hasegawa, Y.; Naganawa, S. Lobar analysis of collapsibility indices to assess functional lung volumes in COPD patients. Int. J. Chronic Obstr. Pulm. Dis. 2014, 9, 1347-1356. [CrossRef]

10. Iwano, S.; Kitano, M.; Matsuo, K.; Kawakami, K.; Koike, W.; Kishimoto, M.; Inoue, T.; Li, Y.; Naganawa, S. Pulmonary lobar volumetry using novel volumetric computer-aided diagnosis and computed tomography. Interact. Cardiovasc. Thorac. Surg. 2013, 17, 59-65. [CrossRef] [PubMed] 
11. Crossley, D.; Renton, M.; Khan, M.; Low, E.V.; Turner, A.M. CT densitometry in emphysema: A systematic review of its clinical utility. Int. J. Chronic Obstr. Pulm. Dis. 2018, 13, 547-563. [CrossRef] [PubMed]

12. Yabuuchi, H.; Matsuo, Y.; Tsukamoto, H.; Horiuchi, T.; Sunami, S.; Kamitani, T.; Jinnouchi, M.; Nagao, M.; Akashi, K.; Honda, $\mathrm{H}$. Evaluation of the extent of ground-glass opacity on high-resolution CT in patients with interstitial pneumonia associated with systemic sclerosis: Comparison between quantitative and qualitative analysis. Clin. Radiol. 2014, 69, 758-764. [CrossRef] [PubMed]

13. Cheng, T.; Li, Y.; Pang, S.; Wan, H.; Shi, G.; Cheng, Q.; Li, Q.; Pan, Z.; Huang, S. Normal lung attenuation distribution and lung volume on computed tomography in a Chinese population. Int. J. Chronic Obstr. Pulm. Dis. 2019, 14, 1657-1668. [CrossRef] [PubMed]

14. Gattinoni, L.; Caironi, P.; Pelosi, P.; Goodman, L.R. What has computed tomography taught us about the acute respiratory distress syndrome? Am. J. Respir. Crit. Care Med. 2001, 164, 1701-1711. [CrossRef] [PubMed]

15. Barile, M.; Hida, T.; Hammer, M.; Hatabu, H. Simple quantitative chest CT for pulmonary edema. Eur. J. Radiol. Open 2020, 7 , 100273. [CrossRef] [PubMed]

16. Kato, S.; Nakamoto, T.; Iizuka, M. Early diagnosis and estimation of pulmonary congestion and edema in patients with left-sided heart diseases from histogram of pulmonary CT number. Chest 1996, 109, 1439-1445. [CrossRef] [PubMed]

17. Narang, N.; Chung, B.; Nguyen, A.; Kalathiya, R.J.; Laffin, L.J.; Holzhauser, L.; Ebong, I.A.; Besser, S.A.; Imamura, T.; Smith, B.A.; et al. Discordance Between Clinical Assessment and Invasive Hemodynamics in Patients With Advanced Heart Failure. J. Card. Fail. 2020, 26, 128-135. [CrossRef] [PubMed]

18. Goetze, J.P.; Bruneau, B.G.; Ramos, H.R.; Ogawa, T.; de Bold, M.K.; de Bold, A.J. Cardiac natriuretic peptides. Nat. Rev. Cardiol. 2020, 17, 698-717. [CrossRef] [PubMed]

19. Callan, P.; Clark, A.L. Right heart catheterisation: Indications and interpretation. Heart 2016, 102, 147-157. [CrossRef] [PubMed]

20. Uriel, N.; Sayer, G.; Imamura, T.; Rodgers, D.; Kim, G.; Raikhelkar, J.; Sarswat, N.; Kalantari, S.; Chung, B.; Nguyen, A.; et al. Relationship Between Noninvasive Assessment of Lung Fluid Volume and Invasively Measured Cardiac Hemodynamics. J. Am. Heart Assoc. 2018, 7, e009175. [CrossRef] [PubMed] 